\title{
Articles
}

\section{Frequency-Dependent Shape Changes of Colloidal Clusters under Transverse Electric Field}

\author{
Ajay Singh Negi,,$^{\ddagger}$ Kheya Sengupta $, \dagger, \S$ and A. K. Sood $*$ \\ Department of Physics, Indian Institute of Science, Bangalore - 560012, India, and Raman \\ Research Institute, Bangalore - 560080, India
}

Received May 11, 2005. In Final Form: August 29, 2005

\begin{abstract}
We have studied clustering of colloidal particles under the influence of an ac electric field as a function of frequency. The field was applied in a direction perpendicular to the confining walls. Two regimes are observed, a low frequency regime where the clusters are isotropic with a local triangular order, as reported earlier in the literature, and a new high-frequency regime where the clusters are highly elongated (anisotropic) with no local order. The crossover from one regime to the other occurs at a critical frequency, $f_{\mathrm{c}}$. The threshold field for the cluster formation, $E_{\mathrm{th}}$, increases with frequency in both the regimes. An increase in the particle size leads to a reduction in both $E_{\text {th }}$ and $f_{\mathrm{c}}$. We present evidence to show that the elongated structures seen at high frequency are related to the field inhomogeneities at imperfections on the conducting surface. We also propose a possible mechanism based on hydrodynamic flow considerations to explain the formation of these clusters.
\end{abstract}

\section{Introduction}

Electric field can be used to control assembly of colloidal particles on the surface of an electrode. ${ }^{1}$ This offers a simple technology to create designed surface architectures, for example, in manufacture of miniaturized biosensors. ${ }^{2}$ Apart from the technical importance, it also has implications in fundamental studies in basic science. ${ }^{3}$ This has led to a spurt of both experimental and theoretical interest in this field in recent years. Electric-field-induced clustering of colloidal particles has been studied in two types of geometries. In one case, both the electrodes are on the same plate and the field is parallel to the confining plates. ${ }^{4-9}$ It is observed that the dielectric particles under parallel electric field will undergo linear aggregation, forming pearl-chains of the particles with the chain axis in the direction of the electric field. ${ }^{4-6}$ These pearl-chains result from the electric-field-induced dipole-dipole interaction between the particles. Pearl chain formation is not restricted to dielectric particles only. Bhatt et. al. ${ }^{10}$ have used metallic nanoparticles to form microwires using

* Corresponding author: asood@physics.iisc.ernet.in.

† Present address: Institute for Medicine and Engineering, University of Pennsylvania, 1080 Vagelos, 3340 Smith Walk, Philadelphia, PA 19104.

$\ddagger$ Indian Institute of Science.

\& Raman Research Institute.

(1) Trau, M.; Saville, D. A.; Aksay, I. A. Science 1996, 272, 706.

(2) Velev, O. D.; Kaler, E. W. Langmuir 1999, 15, 3694.

(3) Zhang, K.; Liu, X. Y. Nature 2004, 429, 739.

(4) Lumsdon, S. O.; Kaler, E. W.; Velev, O. D. Langmuir 2004, 20, 2108.

(5) Fraden, S.; Hurd, A. J.; Meyer, R. B. Phys. Rev. Lett. 1989, 63, 2373

(6) Richetti, F.; Prost, J.; Clark, N. In Physics of Complex and Supermolecular Fluids; Safran, S., Clark, N., Eds.; Wiley: New York, $1987 ;$ p 387.

(7) Hu, Y.; Glass, J. L.; Griffith, A. E. J. Chem. Phys. 1994, 100, 4674 (8) Isambert, H.; Ajdari, A.; Viovy, J. L.; Prost, J. Phys. Rev. Lett. 1997, $78,971$.

(9) Kiriyama, T.; Ozawa, T.; Akimoto, T.; Yoshimura, H.; Mitsui, T. Jpn. J. Appl. Phys. 1997, 36, 7282.

(10) Bhatt, K. H.; Velev, O. D. Langmuir 2004, 20, 467. ac electric fields. Apart from chains, bands of particles have also been observed to form in directions other than the applied field. ${ }^{7-9}$ In the other case, pertinent to the present work, electric field is applied perpendicular to the plates confining the colloidal suspension. $.^{1,6,11-18} \mathrm{Be}-$ yond a threshold applied field, the colloidal particles are seen to form two-dimensional (2-d) isotropic clusters on the confining plates. It is seen that both alternating $(\mathrm{ac})^{1,6,12}$ and direct $(\mathrm{dc})^{12,13}$ electric fields can give rise to such clustering. The mechanism of cluster formation in ac fields has been identified to be of electrohydrodynamic origin,,$^{1,11}$ whereas in dc fields, electro-osmosis is the governing effect. ${ }^{14-16}$ Trau et al. ${ }^{1}$ noted that an increase in the frequency of the applied field results in melting of the clusters. Experiments of Nadal et al. ${ }^{17}$ and Kim et al. ${ }^{18}$ show that the attraction between two particles decreases with an increase in frequency. Sides ${ }^{19}$ extended Trau et al.'s electrohydrodynamic model ${ }^{1,11}$ and showed that the attraction between the particles decreases as the frequency of the applied field is increased. Ristenpart et al. ${ }^{20}$ have presented a scaling analysis to show that attraction between the particles is inversely proportional to the frequency of the applied field. However, to the best of our knowledge, hitherto there are no reports on the effect of frequency on the morphology of the aggregates.

(11) Trau, M.; Saville, D. A.; Aksay, I. A. Langmuir 1997, 13, 6375

(12) Yeh, S. Seul, M. Shraiman, B. Nature 1997, 386, 57.

(13) Bohmer, M. Langmuir 1996, 12, 5747. 6058

Solomentsev, Y.; Bohmer, M.; Anderson, J. Langmuir 1997, 13

(15) Solomentsev, Y.; Guelcher, S.; Bevan, M.; Anderson, J. Langmuir 2000, 16, 9208 .

(16) Guelcher, S.; Solomentsev, Y.; Anderson, J. Powder Technol. 2000, 110, 90 .

(17) Nadal, F.; Argoul, F.; Hanusse, P.; Pouligny, B.; Ajdari, A. Phys. Rev. E 2002, 65, 61409 .

(18) Kim, J.; Guelcher, S.; Garoff, S.; Anderson, J. Adv. Colloid Interface Sci. 2002, 96, 131.

(19) Sides, P. J. Langmuir 2001, 17, 5791.

(20) Ristenpart, W. D.; Aksay, I. A.; Saville, D. A. Phys. Rev. E 2004 $69,21405$. 


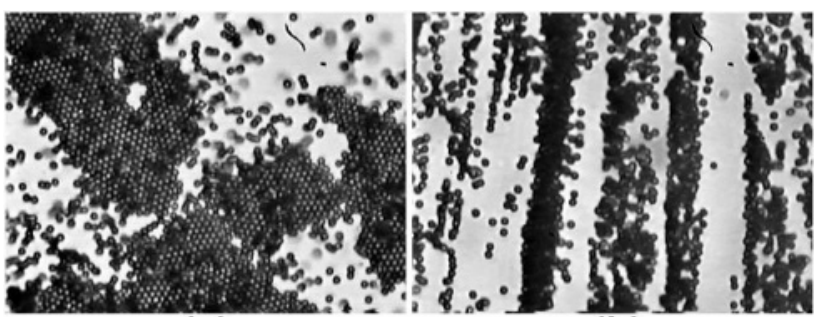

(a)

(b)

Figure 1. Morphology of the clusters depends on the frequency of the applied electric field. (a) Randomly shaped isotropic cluster. Frequency $=1 \mathrm{kHz}$, applied voltage $=3 \mathrm{~V}$. (b) Elongated cluster. Frequency $=100 \mathrm{kHz}$, applied voltage $=6 \mathrm{~V}$. The particles shown here have diameter $2.1 \mu \mathrm{m}$.

Our experiments reported here are done with ac fields perpendicular to the confining plates on an aqueous colloidal dispersion of polystyrene particles. Initially, under a small electric field, most of the particles get confined to a plane parallel to and situated near to one (gravity favors the bottom) of the electrodes. The particles execute a 2-d random walk in this plane. On increasing the field above a threshold, the particles start coming together to form clusters. At low frequencies, the isotropic clusters reported earlier ${ }^{1,6,11-13}$ are observed. At frequencies beyond a critical value, the clusters undergo a drastic change in their morphology. The resulting clusters are highly elongated and seem to locally break the rotational symmetry of the sample geometry. The threshold field for the cluster formation is seen to increase with frequency. To account for this phenomenon, we propose a model based on the phase lag between the polarization of the electric double layer around a particle and the applied electric field, leading to the rotation of the colloidal particles and their coming together.

\section{Experimental Details}

The colloidal suspensions of polystyrene particles with desired volume fractions $(0.1 \%$ for $0.98 \mu \mathrm{m}$ and $0.85 \%$ for $2.1 \mu \mathrm{m})$ were prepared by adding deionized water to the stock suspension (10\%) bought from M/s Bangslabs. To study the effect of electric field, a few drops of the colloidal suspension were sandwiched between two indium - tin oxide (ITO)-coated glass plates, separated by a spacer of $125 \mu \mathrm{m}$ thickness. The ITO plates (sheet resistance of $10 \Omega$ per square) were gifted to us by Prof. N. V. Madhusudana of Liquid Crystal Group of Raman Research Institute, Bangalore, India, who, in turn, had bought these from M/s PPG Industries. The cell was sealed with wax to prevent evaporation of water. A function generator combined with a potentiometer was used to regulate the strength and the sinusoidal frequency of the applied electric field. A Nikon Optiphot2-Pol polarizing microscope (in transmission mode) combined with a CCD video camera was used to image the particles. To obtain the threshold electric field at a given frequency, the voltage was increased from zero in steps of $0.2 \mathrm{~V}$. At each voltage, sufficient time was allowed for the system to reach steady state (indicated by the stabilization of the current).

\section{Results}

3.1. Low-Frequency Results. Upon application of a small electric field of low frequency (less than $3 \mathrm{kHz}$ for particle of diameter $2.1 \mu \mathrm{m}$ ), the colloidal particles are attracted to the conducting plates and undergo a 2-d Brownian motion on the lower plate. ${ }^{6}$ Increasing the strength of the field results in appearance of regions of high and low particle densities and above a certain threshold field $E_{\text {th }}^{\text {iso }}$, the particles start to aggregate into randomly shaped isotropic clusters in which the particles are arranged in a 2-d triangular lattice (Figure 1a). By

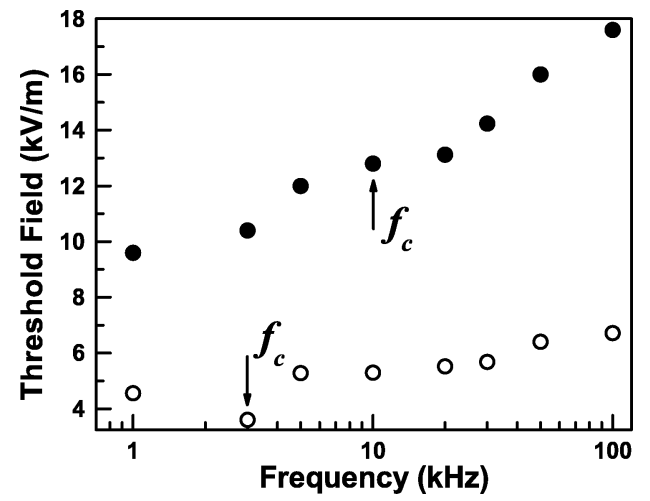

Figure 2. Threshold field increases with frequency for both $2.1 \mu \mathrm{m}$ (open circles) and $0.98 \mu \mathrm{m}$ (filled circles) particles.

changing the focus of the microscope, it can be clearly seen that these clusters are only one particle-layer thick and are thus 2-d. These structures are dynamic; the particles at the edges of the clusters move in and out, and new particles continuously come in to join the clusters. Particles from distances up to tens of micrometers can be seen to migrate directionally toward any given cluster. The velocity of the particles $(\approx 2 \mu \mathrm{m} / \mathrm{s})$ is much larger than that in case of pure diffusion that is observed in the absence of the applied field, and it increases with increase in the strength of the applied field. A steady state is reached when particles get sufficiently depleted from the region around each cluster.

As the frequency is increased, the threshold field, $E_{\text {th }}^{\text {iso }}$, also increases (Figure 2). The isotropic clusters formed at a lower frequency melt when the frequency is increased (keeping the field fixed at the threshold field), but clusters can form again upon subsequently increasing the field above the new threshold field. The clusters are isotropic up to a frequency $\left(f_{\mathrm{c}}\right)$ of $3 \mathrm{kHz}$ for $2.1 \mu \mathrm{m}$ particles and up to $10 \mathrm{kHz}$ for $0.98 \mu \mathrm{m}$ particles.

3.2. High-Frequency Results. Above the critical frequency, $f_{c}$, clusters of a different morphology are formed, as shown in Figure 1b. These are highly elongated structures and are less ordered than the isotropic clusters; in fact they are not strictly 2-d but can be several particles thick. The structures are highly dynamic, and the particles comprising the structures appear to be violently agitated and revolve around each other. The clusters typically range between 3 and 6 particles in width, 2 and 4 particles in thickness, and many $(>20)$ particles long. They are frequently seen to form in pairs or in parallel arrays. Sometimes two elongated clusters are seen to cross each other at an arbitrary angle. Particles are seen to migrate to the elongated clusters with velocities of about $\approx 0.2$ $\mu \mathrm{m} / \mathrm{s}$, about 10 times slower than in the case of the isotropic clusters.

Like the isotropic clusters, the elongated clusters also require a minimum threshold field, $E_{\text {th }}^{\text {elong, for their }}$ formation, which, as with the isotropic clusters, increases with frequency (Figure 2). It can be seen that both $E_{\text {th }}^{\text {iso }}$ and $E_{\text {th }}^{\text {elong }}$ decrease with increasing particle size. A similar trend is observed in particles with a diameter of $4 \mu \mathrm{m}$ (detailed experiments on $4 \mu \mathrm{m}$ could not be performed since these particles were not very Brownian).

3.3. Dynamics of Morphology Change. If a moderately high field is applied at a low frequency (less than $f_{\mathrm{c}}$ ), isotropic clusters are formed (Figure 3a). Now, on increasing the frequency above $f_{c}$, the clusters break up into elongated domains (Figure 3c). The dynamics of this transformation is rather interesting. The isotropic clusters 


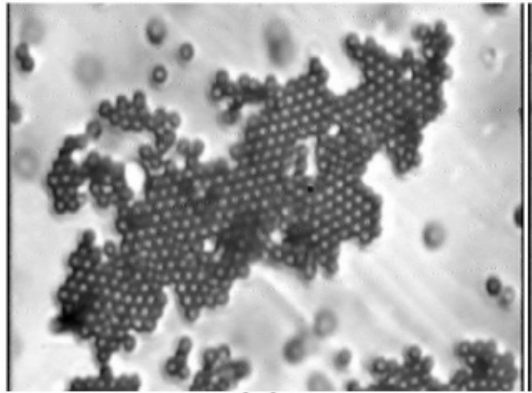

(a)

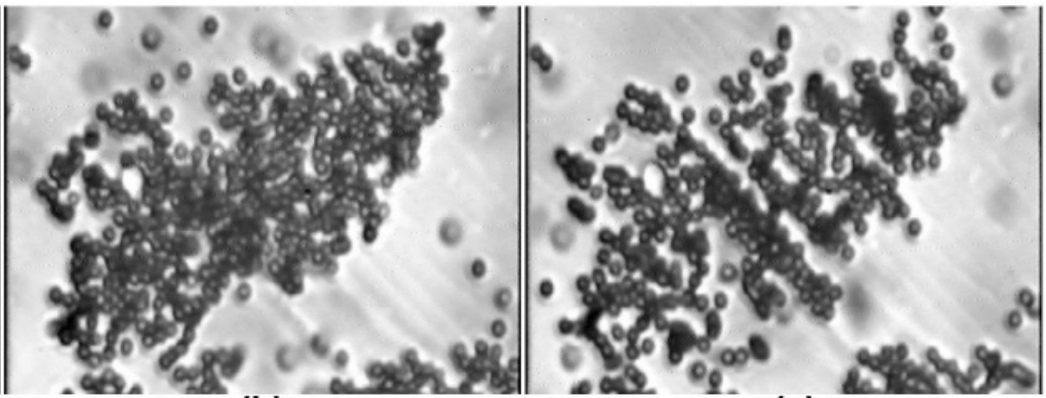

(b)

(c)

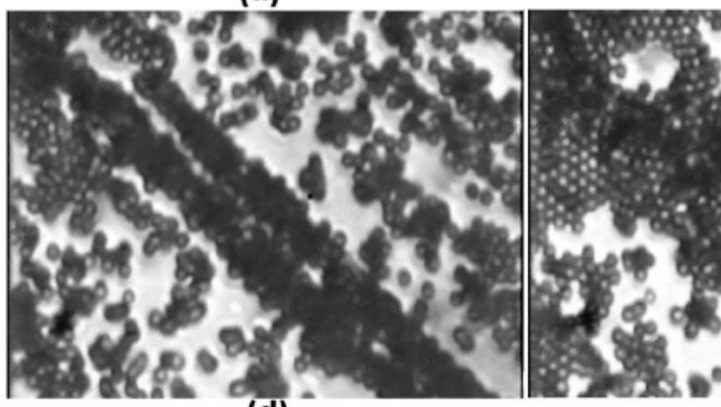

(d)

(b)

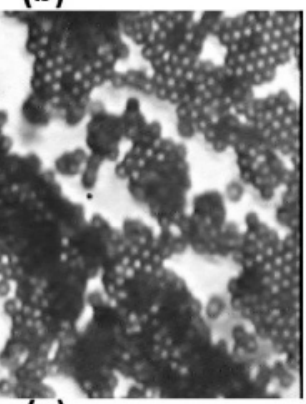

(e)

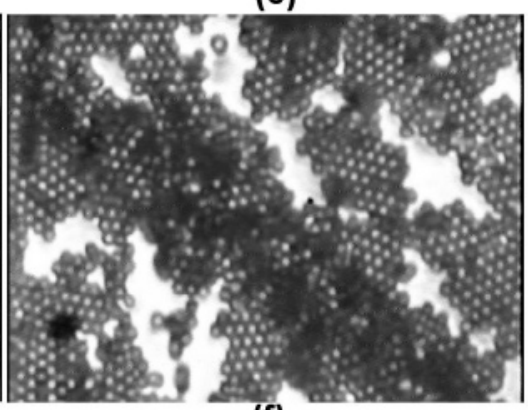

(f)

Figure 3. Morphology of clusters changes with frequency. An isotropic cluster (a) at $f=1 \mathrm{kHz}$ changed into an elongated cluster (c) through an intermediate state (b) after increasing the frequency to $10 \mathrm{kHz}$. Similarly, an isotropic cluster (f) is developed from an elongated cluster (d) as the frequency is lowered from $10 \mathrm{kHz}$ to $1 \mathrm{kHz}$. (e) The intermediate state in the transition. Particle size is $2.1 \mu \mathrm{m}$.

develop "trenches" or "furrows" (Figure 3b) such that particles there seem to repel each other and an array of elongated clusters are formed from the initial isotropic cluster. These "furrows" are seen to develop from some point of irregularity on the edge of the initial isotropic cluster and propagate inward (but not necessarily in every isotropic cluster; some clusters just melt away). On the other hand, if elongated clusters are formed at high frequencies (Figure 3d) and then the frequency is reduced, the space between elongated clusters is filled with particles (Figure 3e), thus destroying the anisotropy of the clusters (Figure 3f).

3.4. Cluster Formation in Inhomogeneous Field. To check our idea that the inhomogeneities in the electric field may be responsible for the elongated structures at high frequency, the conducting ITO layer from a large area on the bottom electrode was etched off. At the interface between the conducting and the nonconducting area, the applied electric field has a discontinuity. At low frequency, as usual, isotropic clusters are observed on the conducting area; no clusters form on the nonconducting area. No special activity is observed near the interface (the line separating the conducting from the nonconducting zones). However, as the frequency is increased above the critical frequency, in addition to the usual elongated structures on the conducting side, chains of particle are observed to form on the nonconducting side near the interface. Particles are seen to migrate from the nonconducting side toward the interface and to arrange themselves in linear chains, up to about 10 particles long and perpendicular to the interface (Figure 4). Most interestingly, the frequency at which these chains begin to appear is the same as the critical frequency of the elongated cluster formation described in Section 3.2.

\section{Discussions}

Our results on the morphology, structure, and dynamics of formation of the isotropic clusters at low frequency described above agree well with experiments reported in

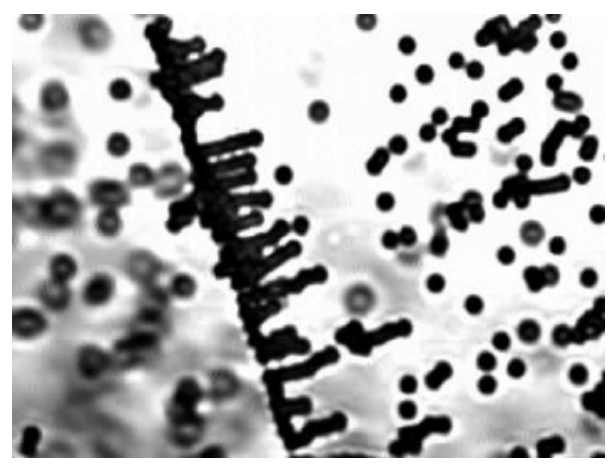

Figure 4. Particles (diameter $=2.1 \mu \mathrm{m}$ ) align themselves perpendicular to the interface, projecting in the nonconducting region (right side) of the electrode above the critical frequency.

the literature. ${ }^{1,6,12}$ Yeh et al. ${ }^{12}$ and later Nadal et al. ${ }^{17}$ used small tracer particles to show that there is a flow field around the colloidal particles. This flow is responsible for the attraction between the two particles. Nadal et al. ${ }^{17}$ have shown that a small particle, placed near a larger particle in the presence of an ac electric field, is attracted toward the larger particle. The smaller particle moves faster as it gets closer to the larger particle. This motion was observed to be slower for higher frequencies of the applied ac field. This shows that the attractive hydrodynamic drag is lesser at higher frequencies and hence indicates that a higher field is required for the clustering of the particles. This observation along with the experiments of Kim et al. ${ }^{18}$ and analysis by Sides $^{19}$ and Ristenpart et al. ${ }^{20}$ support our result that the threshold field increases with frequency.

In the following, we propose a mechanism to understand the formation of the elongated clusters. Our aim is to qualitatively understand the results, rather than to be very rigorous mathematically. In an alternating field, as the field changes direction, the ions in the Debye-Huckel double layer around the particle must effectively move a distance of the order $2\left(a+\kappa^{-1}\right)$, where $a$ is the radius of 


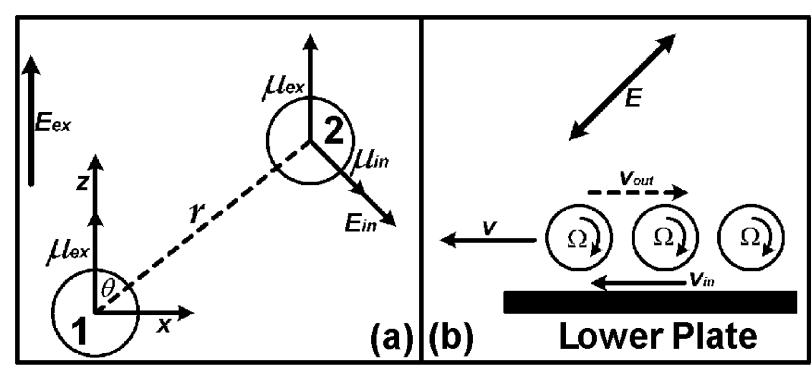

Figure 5. (a) Relative positions of colloidal particles 1 and 2 in suspension. (b) Particle rotation facilitates alignment along a line inclined $45^{\circ}$ to the electric field near the lower plate.

the particle and $\kappa^{-1}$ is the Debye screening length. The time taken for the counterions to diffuse this distance is $\sim\left[2\left(a+\kappa^{-1}\right)\right]^{2} / 6 D$ where $D$ is the diffusion coefficient of the counterions. For the double layer to respond completely to the applied field, it is necessary that the diffusion time be shorter than the oscillation period of the electric field. Therefore, for the frequencies of electric field greater than $6 D /\left[2\left(a+\kappa^{-1}\right)\right]^{2}$, the polarization of the electric double layer around the particles cannot adjust fast enough to the applied field, thereby a phase lag develops between the applied field and the polarization. Considering the counterions to be $K^{+}$ions, $D=1.957 \times 10^{-9} \mathrm{~m}^{2} / \mathrm{s},{ }^{21}$ and taking $\kappa a=43$ (calculated from the conductivity measurements of the suspension), the calculated critical frequency for $2.1 \mu \mathrm{m}$ particle suspension is $2.5 \mathrm{kHz}$ and that for a $0.98 \mu \mathrm{m}$ particle it is $11.7 \mathrm{kHz}$ which are comparable to the observed experimental values of $f_{\mathrm{c}}$. Since $\kappa^{-1} \ll a, f_{\mathrm{c}}^{-1} \sim a^{2} / D$, which is simply the diffusion layer relaxation time often used in colloidal literature. ${ }^{22}$

Following Kiriyama et al., ${ }^{9}$ consider particles 1 and 2 sitting at relative positions at distance $r$, as shown in Figure 5a. Each particle has a dipole moment, $\vec{\mu}_{\mathrm{ex}}$, induced by the external electric field, $\vec{E}_{\text {ex }}$. Considering the angular frequency of the field to be $\omega$ and the phase lag in the polarization to be $\psi$, they are expressed by $\vec{E}_{\text {ex }}=E_{\text {ex } 0} \cos$ $\omega t$ and $\vec{\mu}_{\mathrm{ex}}=\alpha(\omega) E_{\mathrm{ex} 0} \cos (\omega t-\psi)$, where $E_{\mathrm{ex} 0}$ is the amplitude of the external electric field and $\alpha(\omega)$ is the polarizability of the double layer as a function of $\omega$. The dipole moment $\vec{\mu}_{\mathrm{ex}}$ of the particle 1 induces an electric field, $\vec{E}_{\text {in }}$, at the position of particle 2 and thus a dipole moment, $\vec{\mu}_{\text {in }}$, in particle 2 . These are given by $\vec{E}_{\text {in }}=E_{\text {in } 0}$ $\cos (\omega t-\psi)$ and $\vec{\mu}_{\text {in }}=\alpha(\omega) E_{\text {in } 0} \cos (\omega t-2 \psi)$, where $\vec{E}_{\text {in } 0}$ is determined by $\vec{\mu}_{\text {ex }}$. The torque, $\vec{N}$, that rotates particle 2 is given by $\vec{N}=\left(\vec{\mu}_{\text {ex }}+\vec{\mu}_{\text {in }}\right) \times\left(\vec{E}_{\text {ex }}+\vec{E}_{\text {in }}\right)$. On simplifying the above equation, ${ }^{9}$

$$
N=\frac{3 \alpha(\omega)^{2} E_{\mathrm{ex} 0}^{2} \sin 2 \theta(1-\cos 2 \psi)}{16 \pi \epsilon_{0} \epsilon_{\mathrm{r}} r^{3}}
$$

where $\epsilon_{0}$ is the permittivity of vacuum and $\epsilon_{\mathrm{r}}$ is the relative permittivity of water. The angle, $\theta$, and $r$ are defined in Figure 5a. This torque has a maximum amplitude at $\theta=$ $45^{\circ}$. Therefore, a pair of particles aligned along a line inclined at $45^{\circ}$ to the electric field (as shown in the Figure $5 \mathrm{~b})$ rotate in the same direction as indicated by $\Omega$. Taking the no-slip boundary condition, if the centers of the particles are fixed, the rotation will produce water flow as indicated by $v_{\text {in }}$ and $v_{\text {out }}$ in Figure $5 \mathrm{~b}$. It has been seen by Kiriyama et al. ${ }^{9}$ that $v_{\text {out }}=0$. This may be understood

(21) Handbook of Chemistry and Physics, 79th ed.; CRC Press: New York, 1998-1999.

(22) Hunter, R. J. Foundations of Colloid Science; Oxford University Press: New York, 1989; Vol. 2, p 803. by noting that the particles being heavier than water are closer to the lower plate. Therefore, the flow $v_{\text {in }}$ will be confined between the particles and the lower plate. However, the upper plate being far above the particles (almost 60 diameters away), the flow $v_{\text {out }}$ will not be confined. This is a situation in which the liquid is flowing to the left with velocity $v_{\text {in }}$ in a narrow channel. Since the suspension is bounded from all sides (due to the gasket in the sample cell), the liquid is forced to flow to the right in a wider channel with velocity $v_{\text {out }}$. By invoking mass conservation, we see that $v_{\text {out }} \ll v_{\text {in }}$. Therefore, the flow $v_{\text {in }}$ will help in bringing the particles toward each other. For the particles to aggregate, this hydrodynamic force has to overcome the electrostatic repulsive force between the charge stabilized polystyrene particles. We shall now estimate the relative magnitude of these two forces.

A particle rotating in a fluid experiences a retarding viscous torque given by $8 \pi \eta a^{3} \Omega .^{23}$ Equating this torque to eq 1 , we get

$$
\Omega=\frac{3 \alpha(\omega)^{2} E_{\mathrm{ex} 0}^{2} \sin 2 \theta(1-\cos 2 \psi)}{128 \pi^{2} \epsilon_{0} \epsilon_{r} \eta a^{3} r^{3}}
$$

The polarizability of a sphere of permittivity, $\epsilon_{\mathrm{P}}$, in a medium of permittivity $\epsilon_{\mathrm{r}}$ is given by $^{24}$

$$
\alpha=4 \pi a^{3} \epsilon_{0} \epsilon_{\mathrm{r}}\left(\frac{\epsilon_{\mathrm{P}}-\epsilon_{\mathrm{r}}}{\epsilon_{\mathrm{P}}+2 \epsilon_{\mathrm{r}}}\right)
$$

Now, since $\epsilon_{\mathrm{P}} \ll \epsilon_{\mathrm{r}}, \alpha \approx-2 \pi \epsilon_{0} \epsilon_{\mathrm{r}} a^{3}$. Substituting this value of $\alpha$ in eq 2 and taking the values $\sin 2 \theta=1$ and $\cos 2 \psi$ $=-1$ we get the maximum value of $\Omega$ as

$$
\Omega_{\max }=\frac{3 \epsilon_{0} \epsilon_{\mathrm{r}} a^{3} E_{\mathrm{ex} 0}^{2}}{16 \eta r^{3}}
$$

It is known from fluid dynamics ${ }^{23}$ that when a sphere of radius, $a$, rotates at an angular velocity, $\Omega_{\max }$, in an infinite fluid of viscosity, $\eta$, the fluid around it flows with a tangential velocity

$$
v_{\mathrm{T}}=v_{\mathrm{in}}=\frac{a^{3} \Omega_{\max }}{r^{2}}=\frac{3 \epsilon_{0} \epsilon_{\mathrm{r}} a^{6} E_{\mathrm{ex} 0}^{2}}{16 \eta r^{5}}
$$

In Figure $5 \mathrm{~b}$, since $v_{\text {out }}=0$, centers of the particles move in the direction indicated by $v$. We have given a simplistic picture in which $v=v_{\text {in }}$. In the case where $v_{\text {out }}=0, v_{\text {in }}$ will produce a flow field in which the velocity will be $v_{\text {in }}$ near the bottom plate and zero at the top plate (which is at a distance of $125 \mu \mathrm{m})$. Now, another particle which is near the bottom plate will have its center at about $1 \mu \mathrm{m}$ from the bottom plate. The difference in velocities at the two planes which are only $1 \mu \mathrm{m}$ apart will not be significant. We therefore take $v=v_{\text {in }}$. In such a flow field, a neighboring particle will experiences a Stokes drag given by

$$
F_{\text {Stokes }}=6 \pi \eta a v=\frac{9 \pi \epsilon_{0} \epsilon_{\mathrm{r}} a^{7} E_{\mathrm{ex} 0}^{2}}{2 r^{5}}
$$

(23) Landau, L. D.; Lifshitz, E. M. Fluid Mechanics; Pergamon Press: London, 1959.

(24) Pohl, H. A. Dielectrophoresis, the Behaviour of Neutral Matter in Nonuniform Electric Fields; Cambridge University Press: Cambridge, 1978. 


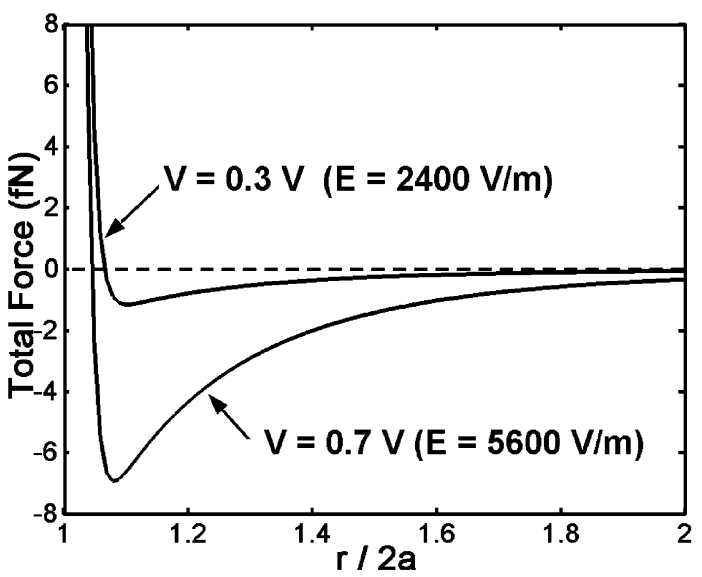

Figure 6. Resultant of the repulsive electrostatic force and the attractive Stokes drag shows an attractive minimum.

Further, the screened Coulomb electrostatic repulsive force is given $b y^{25}$

$$
F_{\text {DLVO }}=\frac{z^{2} e^{2}}{4 \pi \epsilon_{0} \epsilon_{\mathrm{r}}}\left(\frac{\exp (\kappa a)}{1+\kappa a}\right)^{2} \frac{(1+\kappa r)}{r^{2}} \exp (-\kappa r)
$$

where $z e$ is the charge on the polystyrene particles. The resultant of these two forces is given by

$$
F^{\text {Total }}=F_{\text {DLVO }}-F_{\text {Stokes }}
$$

Putting $a=1.05 \mu \mathrm{m}, \kappa a=43$ and $z=3989$, we $\operatorname{plot} F^{\text {Total }}$ as a function of the distance, $r$, between the particles for two values of $E_{\text {ex } 0}$ : 5600 and $2400 \mathrm{~V} / \mathrm{m}$ in Figure 6. For $E_{\text {ex } 0}=5600 \mathrm{~V} / \mathrm{m}$ (which is above the threshold electric field), the minimum in the force is $7 \mathrm{fN}$. Multiplying it with the particle diameter gives an energy, $U \approx 1.5 \times$ $10^{-20} \mathrm{~J}$, which is roughly equal to $4 k_{\mathrm{B}} T$ and hence sufficient for aggregation. On the other hand, for $E_{\text {ex } 0}=2400 \mathrm{~V} / \mathrm{m}$ (which is just below the threshold field), the minimum in the force is $1 \mathrm{fN}$, which is equivalent to $0.5 k_{\mathrm{B}} T$ and hence insufficient for aggregation.

In our experiments, since the electric field is perpendicular to the plane of confinement of the particles, according to eq 1, the above mechanism is not expected to be applicable. To understand the relevance of the above mechanism in the case of the experiments reported here, first consider the case where the ITO is removed from one part of the lower electrode (see Section 3.4). Near the steplike discontinuity, the field is not perpendicular to the electrodes any more (see Figure 7a). Thus, the abovedescribed mechanism comes into play and gives rise to the chain of particles that are perpendicular the interface between the conducting and nonconducting regions. These chains, which are 3-10 particles long, correspond to the chains of similarly rotating particles described above and shown in Figure 4.

Now, even in the case where there are no deliberately introduced inhomogeneities in the conducting surface, imperfections of the coating are always present. AFM measurements show a height variation of the order of 10 $\mathrm{nm}$ on the surface of the ITO plates. If there are microscopic scratches on the electrodes, it is like having a noncon-

(25) Sood, A. K. In Solid State Physics; Ehrenreich, H., Turnbull, D., Eds.; Academic Press: New York, 1991; Vol 45, p 1.

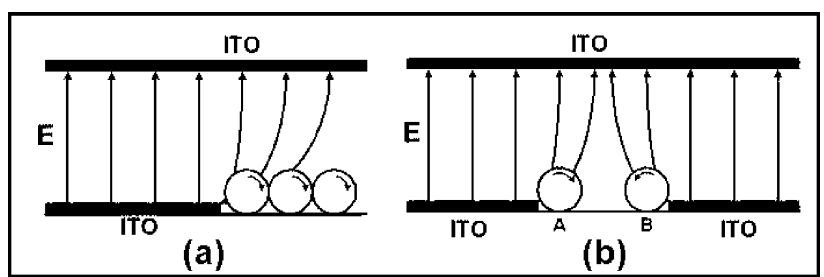

Figure 7. (a) Schematics to show that electric field lines are inclined $45^{\circ}$ to the particles near the interface and lead to the formation of linear chains perpendicular to the interface. (b) The opposite sense of rotation of particles at points A and B will lead to the repulsion between them.

ducting region sandwiched between two conducting regions. The two parallel lines distort the field as shown in Figure 7b. The particles at point A and point B rotate in an opposite sense and repel each other due to the noslip boundary condition. This leads to the formation of two parallel chains of particles that are driven to the defects. The expected velocity of the particles as determined from eq $5(0.14 \mu \mathrm{m} / \mathrm{s}$ for $r=2 a)$, is close to the experimentally determined value of $0.2 \mu \mathrm{m} / \mathrm{s}$.

The development of furrows and trenches within the low-frequency isotropic clusters (Figure $3 \mathrm{~b}$ ) on increasing the frequency, is also consistent with the above explanation. At low frequencies, the cluster formation is driven by hydrodynamic flow due to the external electric field and is not affected by small inhomogeneities. However, at high frequencies, the cluster formation takes place only at points of inhomogeneities. If the microscopic scratches described before happen to run underneath an isotropic cluster, upon increasing the frequency beyond $f_{\mathrm{c}}$, they become sites for particle repulsion. The same arguments also explain why the elongated clusters formed at higher frequencies are converted into isotropic ones on reducing the frequency. The fact that, if the clusters are removed, they appear again at the same positions and with the same orientations strengthens our argument that the surface inhomogeneity is crucial for the formation of anisotropic clusters.

In summary, we have presented a systematic study of frequency dependence of the phenomena of aggregation of colloidal particles in an ac electric field. We have observed that the morphology of the clusters depends on the frequency. The low-frequency isotropic clusters can be explained in terms of electro-hydrodynamic effects..$^{1,11}$ The apparent spontaneous breaking of rotational symmetry by the formation of elongated domains seen at high frequencies is explained in terms of rotation of particles due to a phase lag between the polarization of the electric double layer around a particle and the applied electric field that arises because of inhomogeneities of the conducting surface. The experiments reported here should be useful in developing techniques to manufacture colloidal aggregates of desired morphology by suitably patterning the electrodes. ${ }^{26}$

Acknowledgment. We thank Prof.N.V.Madhusudana for providing us the ITO plates. Debjani's help in photolithography is gratefully acknowledged. A.K.S. thanks the Department of Science and Technology, India and A.S.N. thanks CSIR for financial support.

\section{LA051258B}

(26) van Blaaderen, A.; Ruel, R.; Wiltzius, P. Nature 1997, 385, 321. 